\title{
3-D Polymeric Microfluidic devices for BioMOEMS applications.
}

\author{
${ }^{\mathrm{a} F}$. J. Blanco,${ }^{\mathrm{a} J}$. Berganzo, ${ }^{\mathrm{a} J}$. Garcia, ${ }^{\mathrm{a} K}$. Mayora, ${ }^{\mathrm{b}}$ A. Calle and ${ }^{\mathrm{b}}$ L. M. Lechuga.
}

\author{
Ikerlan S. Coop. Microsystem Department. P JJM. Arizmendiarrieta, 2.20500.Mondragon, Gipuzkoa \\ (Spain) \\ *fjblanco@ikerlan.es \\ ${ }^{b}$ Biosensors Group. Microelectronics National Centre (CNM).CSIC. E-28760 Tres Cantos, Madrid \\ (Spain)
}

\begin{abstract}
This paper describes the fabrication, packaging and characterization of novel multilayer polymer microfluidic systems fabricated by a CMOS compatible process. These microfluidic devices were specially designed for BioMOEMS applications. Embedded multilayer rectangular smooth and uniform microchannels, 50 to $150 \mu \mathrm{m}$ wide and $18 \mu \mathrm{m}$ deep were studied. Steady-state flow rates and pressure driven flow control were measured in the laminar flow regime. Flow rates ranging from 1 to $100 \mu \mathrm{l} / \mathrm{min}$, at pressure drop ranging from 10 to $600 \mathrm{kPa}$, were obtained. These flow rates yield Reynolds numbers $\left(R_{e}\right)$ up to 20 . Results indicate that the experimental $R_{e}$ and the flow friction coefficient (f) are in good agreement with the laminar flow theory. These experimental results facilitate the future designs of different microfluidic devices designed by using classical fluidic theory.

We also present two different methods developed for macro/microfluidic packaging in order to connect these microfluidic devices to the macroscopic world. The microsystem packaging can withstand pressure drops up from 500 to $2000 \mathrm{kPa}$ with any liquid leakage.
\end{abstract}

Keywords: Microfluidics, BioMOEMS, 3-D Polymer Microchannels, Microfluidics packaging, Flow characterization.

\section{INTRODUCTION}

The advance of micro-optical-electro-mechanical-systems (MOEMS) in the field of Microfluidics has opened a wide field of possibilities to improve the sensivity and selectivity of analytical devices. Improvements in overall analytical performance can be achieved by minimizing the scale on which the analysis is performed. The reduction in the size of the analytical device has other advantages including fast analysis, less sample amount, and smaller size. Also, the possibility to integrate different type of sensors, CMOS circuits, micromechanical and microfluidic components in the same manufacturing cycle, reduce the cost of the final device. These strategies have been developed into the concept of Micro Total (bio-chemical) Analysis Systems ( $\mu$ TAS) also called Lab-on-a-chip devices [1-3].

The rapid development of these microfluidic devices has generated a great interest of the Biotechnology community. Using the power of Silicon ICłs microfabrication and novel MOEMS technologies [2,4], microfluidic devices for bioanalytical applications have been developed [3]. Researchers have been able to create microfluidic structures that offer the prospects of precision fluid delivery to targeted locations via controllable flow paths. Based on these principles and technologies Bio-MOEMS devices such as capillary electrophoresis, DNA amplification (PCR), bio-chemical analysis systems, microfluidic drug delivery devices, among others [2,3], have been developed. All these Microsystems require the use of microfluidic channels for liquid or gas delivery, bio-chemical reactions etc.

Precise manipulation of fluid flow through these microchannels is a key step in the analytical performance of these devices [5]. The characteristic length scales that govern the energy and momentum transfer between microfluidic channels and their environments are typically of the order of microns. It is expected that the fluid flow phenomena in 
microchannels are somewhat different from the conventional theory applied to microchannels. A previous modelization of the liquid flow through such microdevices will be critical for the final performance of any Biochip device [5].

Based on the literature survey it seems that only a few papers deal with liquid flow in microchannels [6-18] with different results :

- Wilding et al. (1994) observed increase in pressure drop above theoretical predictions at the high flow rates in straight glass-capped silicon microchannels. They attributed it to inertial losses and entrance length effects which are not included in the theory.

- Peng et al. (1995), Peng and Peterson (1996), and Xu et al. (1999) observed increase/decrease of friction factor/pressure gradient compared to conventional theory. They attributed this to an early onset of laminar-toturbulent flow transition. They found geometric dependence on flow friction factor for different hydraulic diameters and aspect-ratios.

- Pfhaler et al (1990,1991), Papautsky et al. (1999), Mala and Li (1999), Qu el al. (2000), Li (2001), Ren et al. (2001) and Guo and $\mathrm{Li}$ (2003) assumed that the deviation would originate from surface phenomena such as surface roughness, electrokinetic forces, temperature effects and microcirculation near the wall. Most results indicate that the friction factor/pressure gradient could increase due to surface phenomena.effects

- Flockhart et al. (1998), Xu et al (2000), Sharp (2001) and Judy et al. (2002) insisted that the friction factor/pressure gradient results are the same as the values predicted by conventional theory. They claimed that the deviations, which other researchers had observed, might have originated from measurement error of channel size, experimental uncertainties and overlooking of the entrance effect.

The microchannels used in the above studies were manufactured with different materials and fabrication methods: (i) traditional machining of stainless steel and aluminium process [7,8,11] (ii) Silicon and Pyrex bulk and surface micromachining process $[5,6,10,12,14]$ or (iii) polymer micromoulding technologies $(16,17)$. The size $(1-500$ micron wide, 1-100 microns depth), geometries (circular, trapezoidal, rectangular, square), and surface roughness (from 1 to 5\%) were different. These studies were performed under different flow conditions and experimental set-up. In addition, different macro/microfluidic connections were used.

It should be noted that the materials and fabrication process used in above experiments were not designed for BioMOEMS applications. Also, they are not fabricated by CMOS compatible process, which make difficult the future integration of this type of technologies with microelectronics devices in a single chip. However, the microchannels tested in our study were fabricated by a novel CMOS compatible fabrication processes that will allow the integration of different technologies onto a single substrate [19-21].

Another important hurdle that still needs to be performed in microfluidic studies is the connection and packaging between the microfluidic components, and the macro-environment. This task is difficult because liquid samples and reagents are typically transferred in quantities of microliters to milliliters (or even liters) whereas microfluidic devices consume only nanoliters (nL) or picoliters of samples due to the size of reaction chambers and channels. This challenge is often ignored in research laboratories, because skilled personnel operate the device and the mismatch between macroand micro-environments can be avoided. However, it must be addressed prior to commercial success of any microfluidic device. For these reasons the macro-micro interface of the devices must be included in the microfluidic characterization studies. Standardizing interfaces for microfluidic devices to accept macro-samples is important to the future of the field as well as to the commercial success of the technology [22-24]. Though there a number of groups attempting to standardize interconnects and microfluidic devices in general [24], their effectiveness has yet to be truly assets or seen industry-wide, and it will be years before it can be truly measured. We do think an acceptable, agreeable standard interface should exist for each type of application, though it is probably not applicable to all microfluidic devices.

Based on the foregoing discussion, it is necessary to further study the flow phenomena and the macro-micro interfaces during the development of a new technology.

This paper presents the fabrication and flow characterization of novel CMOS compatible microfluidic devices specially designed for BioMOEMS applications. In addition, different packages for macro-microfluidic interfaces are presented. A flow characterization including this important task has not been reported before in microfluidic characterization studies. 


\section{MICROCHANNEL FABRICATION}

The novel micro-fabrication technique developed for the fabrication of the microfluidic devices is based on an adhesive bonding process and releasing steps of two photolithographic patterned SU-8 polymeric resist layers on separate wafers . This technology is described in detail elsewhere [19-21]. Firstly, two wafers were spin coated with SU-8 photoresist. The bottom wafer could be silicon, silicon processed or a Pyrex wafer and the top one is a Pyrex wafer or a Kapton film glued onto a Pyrex wafer. After spin coating, a dry step (soft bake) was done at $90 \mathrm{C}$ in a progressive ramp .The UV exposure was conducted on a Karl Suss MA6/BA6 aligner. The intensity of this aligner was $10 \mathrm{~mW} / \mathrm{cm}^{2}$ at $365 \mathrm{~nm}$. The wafers were exposed for 30-35 seconds depending on the thickness of the layer. The post exposure baking was also performed in a ramp way at $90 \mathrm{C}$ for a period of time determined by the thickness of the material. Finally, the wafers were developed in propylene glycol methyl ether acetate (PGMEA) during three minutes. For the silicon wafer, development was performed by immersion with slight agitation. In the case of the SU8 layer on Kapton remains undeveloped.after finishing the bonding process.

Once the 2-D SU-8 layers have been generated by the photolithography processes, the patterned wafers were bonded together to form 3-D microchannel networks between both wafers. Subsequently, the alignment is carried out (the 150 $\mu \mathrm{m}$ thickness of the film guarantees enough transparency to see through the Kapton-Pyrex stack the undeveloped microstructures). The wafer-level bonding protocol have been established using a commercial bond equipment, a Karl Suss SB6 Bonder. Firstly, the wafers were loaded into the bond chamber. The chamber was evacuated to $10^{-3} \mathrm{mbar}$. Prior to contact, two heaters (bottom and top) started to heat uniformly the wafers at a temperature of $90 \mathrm{C}$ for three minutes, to enable solvent evaporation and prevent any void formation. Afterwards, the wafers were brought into contact and the two heaters heat the wafers at a temperature of 100-110 C, and applying a pressure of $300 \mathrm{KPa}$ for 30 minutes.

After the bonding process the SU8 stack is detached from the Kapton film and developed, and is ready for starting a new cycle . A schematic of the whole process is shown in Figure 1.

SU8 photopatterned on Silicon

Two 8 layers, bonded together

Two SU8 layers bonded together, after releasing the Kapton

Three SU8 layers between wafers

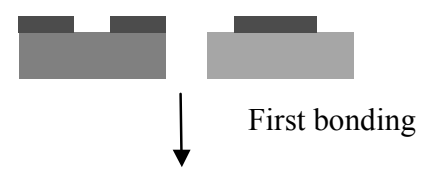

SU8 photopatterned on Kapton

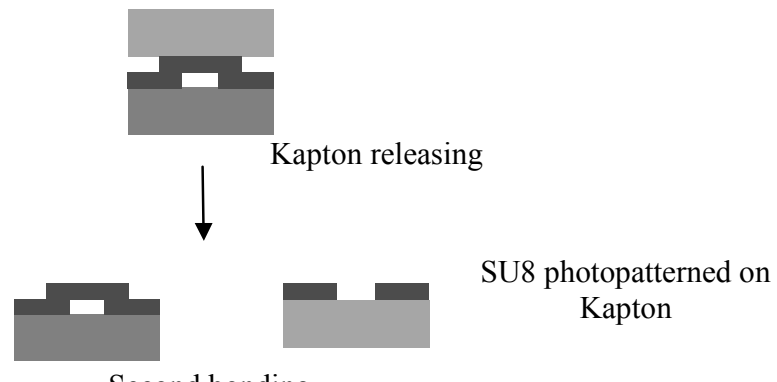

Second bonding

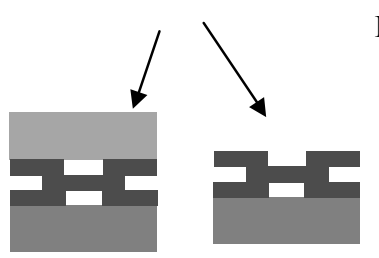

Kapton releasing

Figure 1. Fabrication process of SU8 multilevel microfluidic channels 


\section{MICROFLUIDIC PACKAGING}

We have developed two types of macro-microfluidic packages for connecting the microfluidic systems to the outside. The systems are designed for BioMOEMS applications and should integrate in the future microfluidic, CMOS and Integrated Optical (IO) devices.

An integrated interconnects approach and a modular interface between the chip and the macro-scale connections were tested.

\subsubsection{Integrated lateral connections}

In order to fabricate low cost and robust integrated macro-microfluidic connections a time-pressure dispensing process [24] has been used. The dispensing process is based on surface mount technology (SMT) widely used in electronics assembly industry, in which to secure a component to a board, a minute volume of surface mount adhesive (SMA) is required to be delivered between pads with a typical distance of $500 \mu \mathrm{m}$.

Due to the nature of the SU-8 photolithographic-bonding multilayer process, lateral inlets and outlets can be realized to be connected to the outside world. These microchannels were connected laterally using standard tubing (see Figure 2). The external tubing was glued to the lateral connections by dispensing a SMA. We were used a glue epoxy (Loctite 3609). The bond strength of the glue was $30 \mathrm{MPa}$. We were make use of standard SMT equipment (Dr. Tresky 3002) to align the chip and dispense, in a precisely controlled manner, the fluid epoxy to seal the standard tubing to the microchannel ports.

Using the lateral approach we have obtained a maximum field of view of the microfluidic network. A schematic of a macro-microfluidic packaging is showed in Figure 3.

External tube Epoxy

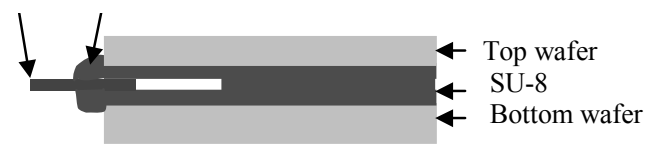

Figure 2. A squematic of the lateral connections using a fluid epoxy. The epoxy is put around the inlet and outlets using a time-dispensing process equipment.

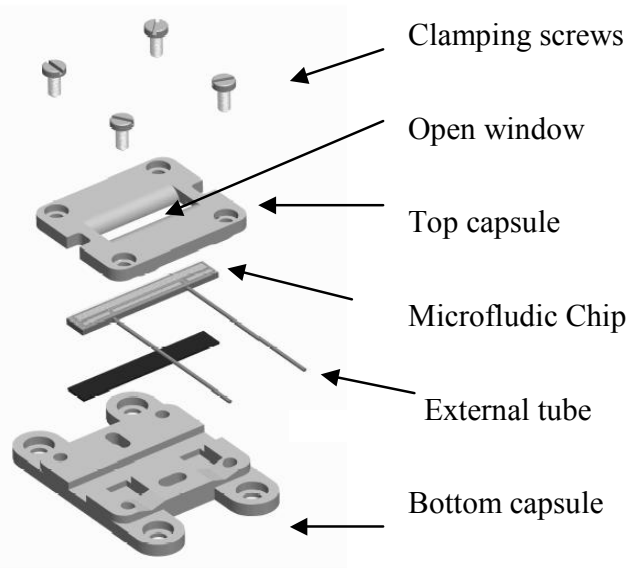

Figure 3. A squematic of the device after its packaging.

\subsubsection{Modular connections}

Recently, research groups have used interfaces between a chip and the macro-scale tools which can be achieved by using a separate module $[26,27,28]$. By this way, several interconnections, including the optical, electrical and microfluidic parts can be integrated in a single package. These modular interfaces are, for the most part, independent of the material the device is fabricated. The macro-tools can include capillaries, tubes, syringes, optical fibers and electrical connections. These tools may be connected to the chip through a large housing that can be treated as a module. This arrangement is typically applied to devices with a small distance between ports and for high throughput analysis, such as DNA analysis [27].

In our approach we have the advantage that the input and the output of the microchannels will be kept in contact with the outside world by using the SU8 Kapton releasing step (see Figure 1) [22]. This avoids the slow drilling or 
etching steps of the cover, radically simplifying the packaging. Hence, a plastic capsule with external flexible tubes and an O-ring (one $\mathrm{mm}$ internal diameter, two $\mathrm{mm}$ outside diameter) per reservoir are enough to insert liquids into channels as can be seen in Figure 4. All the pieces of the packaging are aligned and held together by screws without adhesive, allowing an easy replacement of the devices.
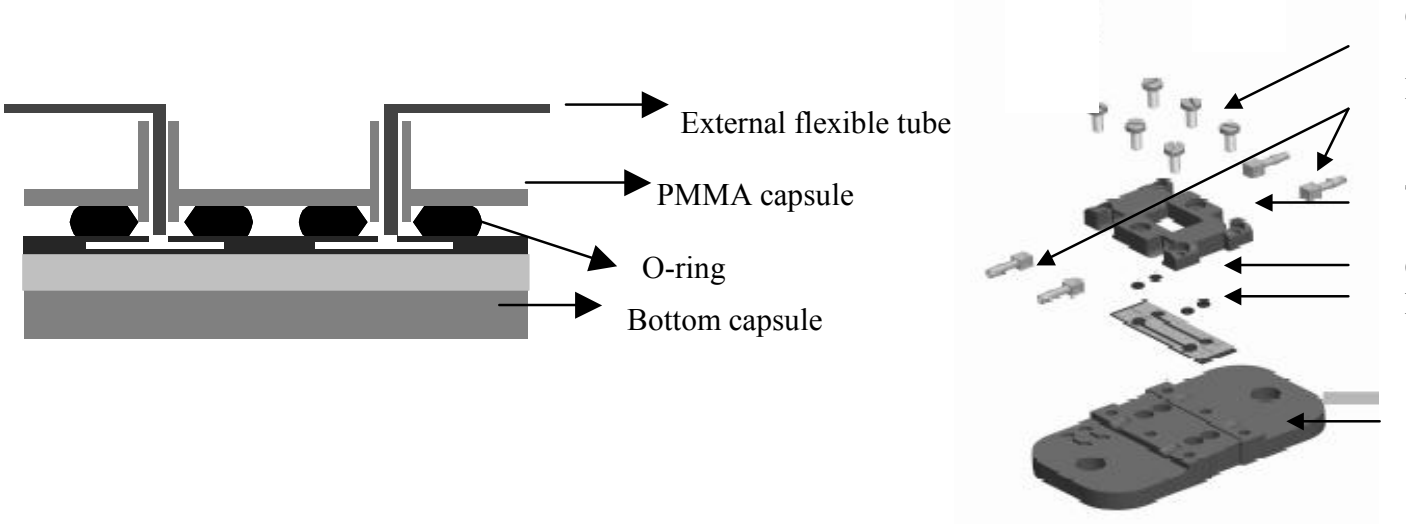

Clamping screws

External tubes

Top capsule

O-rings

Microfluidic Chip

Bottom capsule

Figure 4. On the left, there is a schematic of the macro-microfluidic packaging where the O-rings (there is one per reservoir) are put around the inlets and the outlets avoiding liquid leakage when it is inserted in the channel across the external flexible tube. A Teflon or PMMA top capsule is screwed to the bottom platform board to seal the system. On the right, it is shown a schematic of the packaging prototyping.

\section{CHARACTERIZATION OF FLUID FLOW}

The experimental set-up for the flow characterization was analogous to other microfluidic characterization studies $[6,10,11]$. Precision syringes and a pressure driven system were used to delivery the liquid to the microfluidic chip. The pressure driven system used compressed air to drive the fluid through the system. The air pressure was regulated by a pressure-time dispensing regulator (EFD Ultra 1400 Series). The two types of different macro-microfluidic connections were checked. Deionized (DI) water was used as the working fluid. The experiments were conducted at $21 \int \mathrm{C}$ in a cleanroom environment. A pressure differential transductor (Valydine DP44, Valydine USA) was used to measure the pressure drop between the microchannel inlet and outlet. Mass flow meters were connected to the outlet for flow rates measurements. Two types of mass flow sensors (based on MEMS technology) specific for low flow rate applications were used. From $100 \mathrm{~nL} / \mathrm{min}$ to $6000 \mathrm{~nL} / \mathrm{min}$ a Sensirion SLG-1430-150 was used. Liquid flow rates above 10000 $\mathrm{nL} / \mathrm{min}$ were measured with a Sensirion ASL-1430-16. These flow rates were compared with the ones measured by collecting the liquid from the outlet tube in a specified time interval. The difference between the two flow rates was found less than $\pm 1 \%$.

For the steady state flow measurements, the pressure-driven system was chosen. This pressure-driven system was the fast one to reach the steady state. The pressure injection systems were set to maintain a constant pressure drop across the microchannel, and the flow rate at the outlet of the microchannel was measured. A steady state was reached when the readings of the pressure drop did not change for at least 5 minutes.

Also, the high precision liquid mass flow meters allow us measure the flow response of the systems to switching pressures. This is an important step in order to perform kinetic and diffusion flow studies during the same measurement [27].

The measurements were analyzed and compared with classical flow theory. The parameters used in the study are listed below:

- $\quad$ the hydraulic diameter $\left(D_{h}\right)$, defined as four times the cross-sectional area divided by the wetted perimeter.

- $\quad$ the Reynolds number $\left(\mathrm{R}_{\mathrm{e}}\right)$ is defined as 


$$
\operatorname{Re}=\rho V_{a v e} D_{h} / \mu
$$

where $\mathrm{V}_{\text {ave }}$ is the average velocity of the fluid, $\mu$ the dynamic viscosity and $\mathrm{D}_{\mathrm{h}}$ the hydraulic diameter, respectively.

- the friction factor of a channel is defined by:

$$
\mathrm{f}=\mathrm{k} / \mathrm{Re}
$$

and it can be calculate from the Darcy-Weisbach formula:

$$
\Delta P=\frac{\rho L f V_{a v e}^{2}}{2 D_{h}}
$$

where $V_{\text {ave }}$ is the average velocity of the fluid, $\rho$ the fluid density, $D_{h}$ the hydraulic diameter, $L$ the channel length and $f$ the friction factor, respectively.

\section{EXPERIMENTAL RESULTS AND DISCUSSION}

\subsection{Fabrication and packaging}

Rectangular embedded microchannels have been fabricated using the photolithographic-bonding process. For the flow characterization experiments, $1 \mathrm{~cm}$ length and $18 \mu \mathrm{m}$ height channels were manufactured. The width ranged from $50 \mu \mathrm{m}$ to $150 \mu \mathrm{m}$. These geometries yield $\mathrm{D}_{\mathrm{h}}$ from 28 to $35 \mu \mathrm{m}$. Fig. 5 shows low vacuum scanning-electron-micrographs (SEM) of the microchannels cross-sections. Smooth and uniform rectangular embedded microchannels have been successfully fabricated. It can be observed that the process let the dicing of the devices without detachment or partial deformation of them, allowing us accurately measured channel sizes and geometries by using confocal microscopy and SEM techniques [19]. The errors originated from measurement of channel size and geometries can be ignored [18]. It was not well documented the microchannel dimensions presented in other microfluidic studies [6,7]. The pressure drop in a microchannel is inversely proportional to fourth power of the $\mathrm{D}_{\mathrm{h}}$ of the channel, so an inaccuracy of $5 \%$ in measuring $D_{h}$ can bias flow resistance results by $20 \%$, enough to explain the majority of the discrepancies between the conventional macroscopic flow resistance predictions and the observed values $[10,18]$.
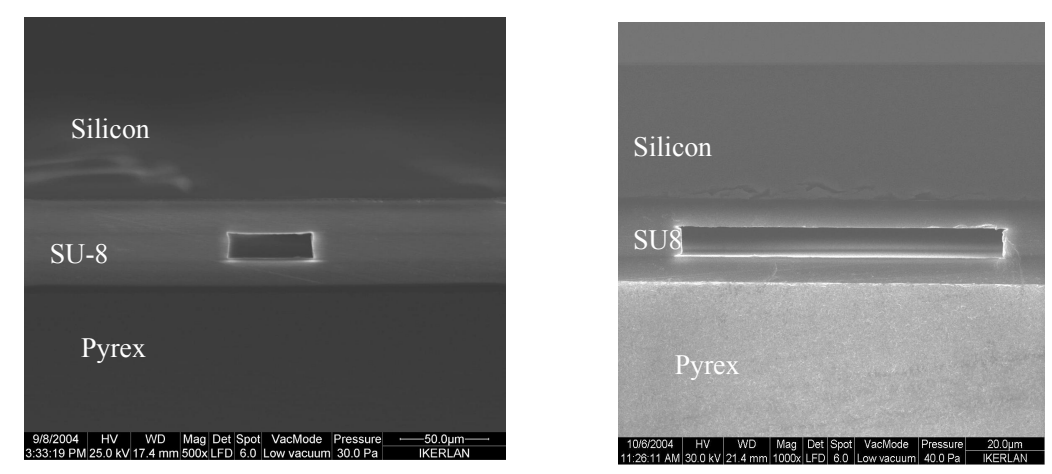

Figures 5. SEM photographs of the microchannel cross sections. On the left, a $50 \mu \mathrm{m}$ wide and $18 \mu \mathrm{m}$ height SU8 channel. On the right, a $150 \mu \mathrm{m}$ wide and $18 \mu \mathrm{m}$ height SU8 microchannel is shown. 
To connect these microchannels to the outside, the integrated and the modular approaches were tested. Figure 6 shows the lateral connection of the devices using the thermal epoxy. It is shown the external tubing sealed by the epoxy. These lateral connections can withstand pressure drop up to $2000 \mathrm{kPa}$ without any liquid leakage. Work is carried on in order to improve this technology. Our goals point at to dead volumes reduction. The flow characterization studies were performed using this packaging technology.

For the modular connections (Figure 7) the packaging can withstand pressure drops up to $500 \mathrm{kPa}$ without any liquid leakage. This system let an easy replacement (plug and play) of the external connections. Further work is on going to increase the range of working pressures using new designs and high precision components, such as planar o-rings and special external tubing.

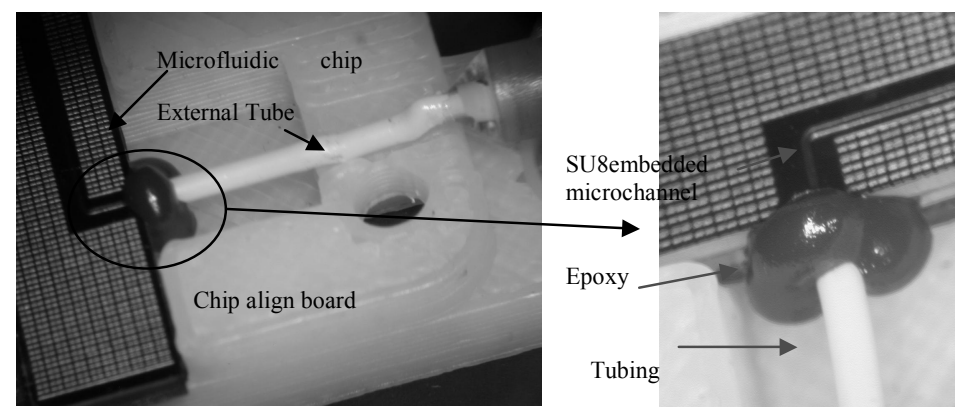

Figure 6. Photographs of the integrated lateral connections. The epoxy (red) was dispensed by a SMT tool.
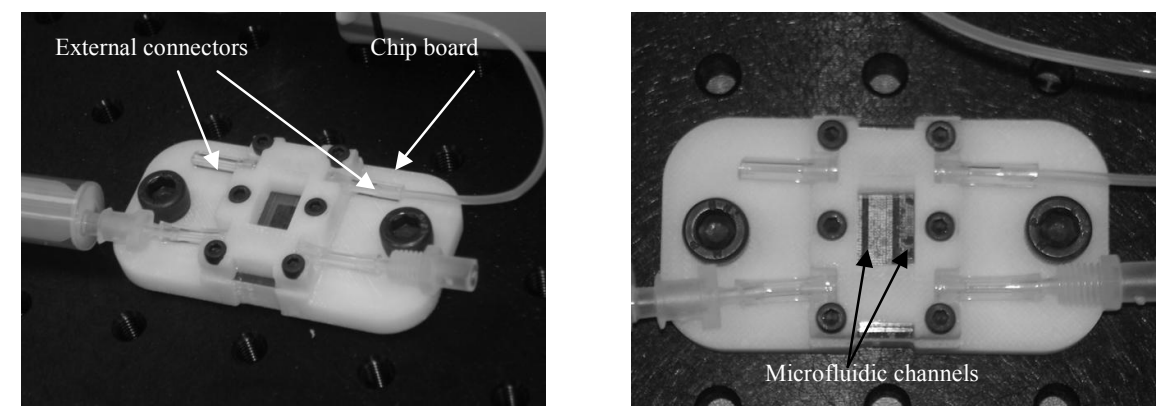

Figure 7. Photographs of the modular packaging connections. Different types of (plug and playßconnectors are shown.

\subsection{Pressure drop and flow rate}

The experiments were conducted with various working pressures, which yield $R e$ up to 20 for microchannels with a hydraulic diameter of about $30 \mu \mathrm{m}$. To make a more accurate measurement, the inlet and outlet pressure losses were deducted before the friction factor calculation. The present pressure drop (P) should be the pressure drop of the DI water through a microchannel of length $1 \mathrm{~cm}$ without inlet and outlet losses. The pressure drop across the external system (tubing) was calculated to be less than $1 \%$ of the total pressure drop through the microchannel.

In Figure 8 and 9, the flow rate measurements for 50 and $150 \mu \mathrm{m}$ width microchannels are plotted as a function of pressure drop. The result exhibit a linear behavior which is in excellent agreement with the traditional conventional theory where flow rate is proportional to pressure drop. However, the relationship seems to deviate when pressure drop increases. These trends are similar to those obtained in other studies $[6,10,18]$ We have attributed this trend to inertial losses and entrance length effects that are not included in the theory.

The friction factors obtained were plotted against $R_{e}$ as shown in Figure 10 and 11. The data exhibits a linear behavior with $\mathrm{R}_{\mathrm{e}}$ indicating the existence of a laminar flow pattern. The power dependence on Re were obtained. Namely, the slope of the distribution. These results give a nearly minus one slope (-1.04 for $50 \mu \mathrm{m}$ width channel and $-0,96$ for 150 
$\mu \mathrm{m}$ width channel). In fact, in the conventional theory for a rectangular channel $50 \mu \mathrm{m}$ width and $20 \mu \mathrm{m}$ height (aspect ratio 0.36 ), the friction factor of a laminar fully developed flow is given by $f=71.5 / R e$, while for a $150 \mu \mathrm{m}$ width and 18 $\mu \mathrm{m}$ height (aspect ratio 0.12) $f=84.55 / R e$. The present values roughly agree with conventional theory.

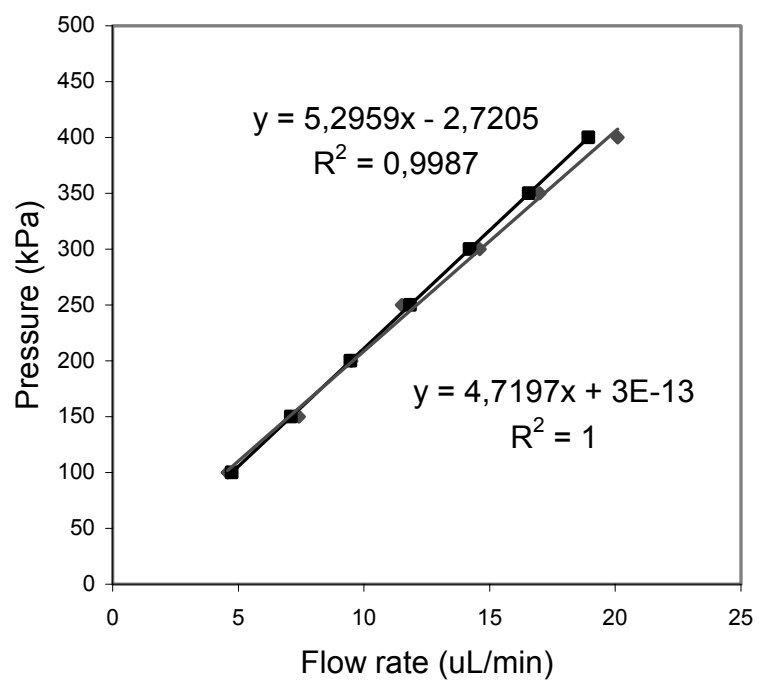

Figure 8. DI water experimental flow rates (red line) at different pressures in a $50 \mu \mathrm{m}$ width and $18 \mu \mathrm{m}$ microchannel compared with conventional fluid theory (black line).

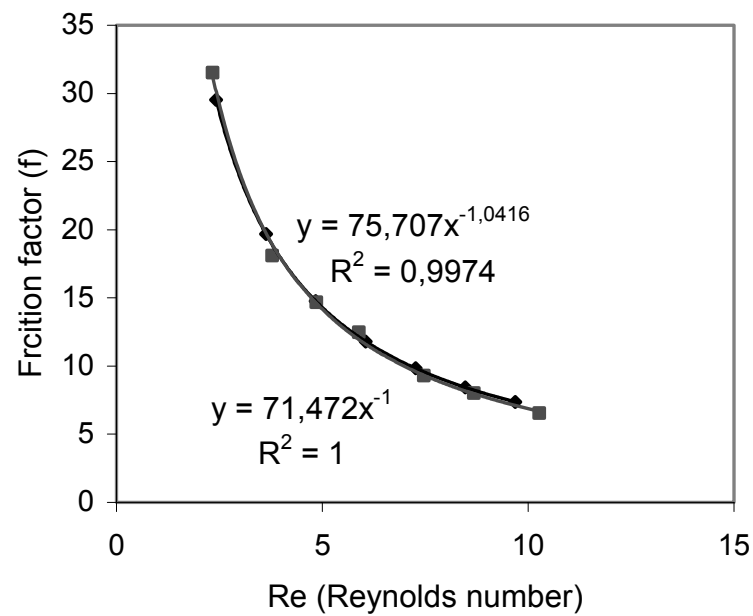

Figure 10. Experimental f vs. Re (red line) $50 \mu \mathrm{m}$ width and 18 $\mu \mathrm{m}$ microchannel compared with laminar flow theory (black line).

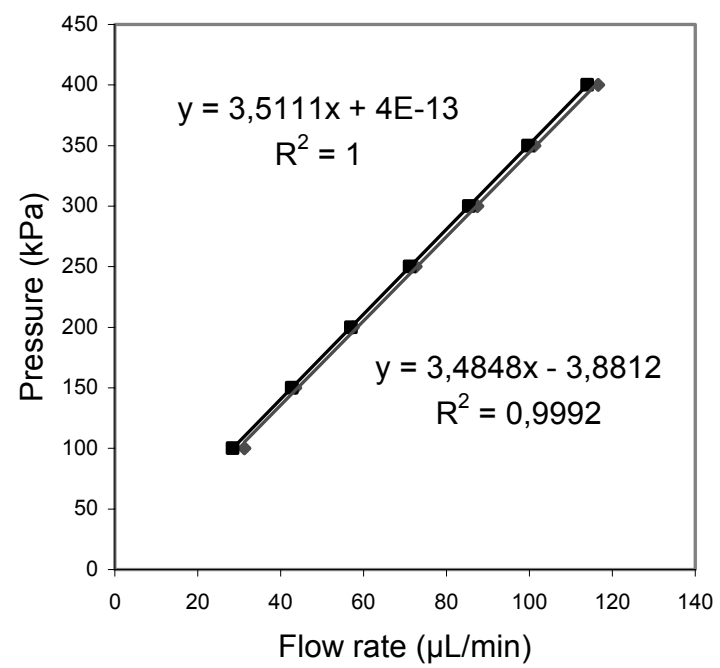

Figure 9. DI water experimental flow rates (red line) at different pressures in a $150 \mu \mathrm{m}$ width and $18 \mu \mathrm{m}$ microchannel compared with conventional fluid theory (black line).

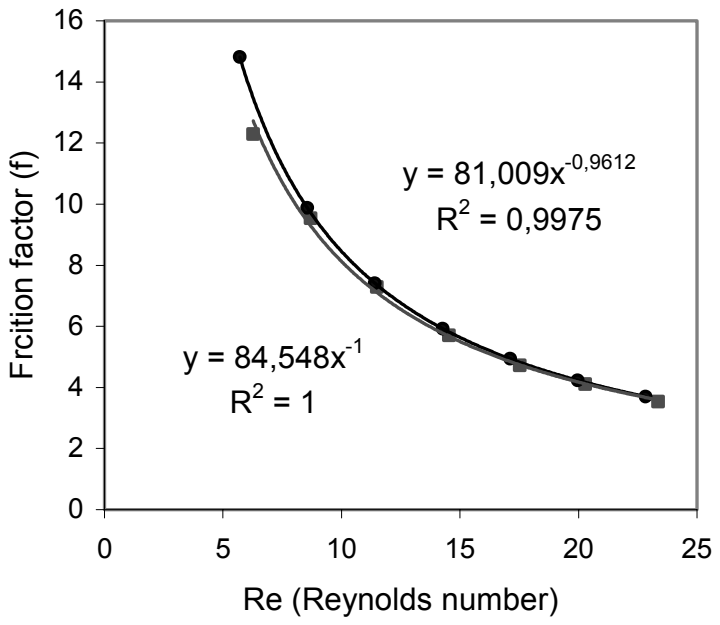

Figure 11. Experimental f vs. Re (red line) $150 \mu \mathrm{m}$ width and 18 $\mu \mathrm{m}$ height microchannel compared with laminar flow theory (black line).

Switching pressure experiments using the pressure driven-system were conducted to check the system flow response. Low working pressures were used to control very slow flows. Figure 12 shows an example of flow control using this approach in a $150 \mu \mathrm{m}$ width and $18 \mu \mathrm{m}$ height microchannel. Flow rates from $1000 \mathrm{~nL} / \mathrm{min}$ to $2000 \mathrm{~nL} / \mathrm{min}$ are controlled at working pressures from $10 \mathrm{kPa}(0.1 \mathrm{Bar})$ to $20 \mathrm{kPa}(0.2 \mathrm{Bar})$. The time to reach the steady-state is around 1 minute. By using other injection systems, such as precision syringes the time to reach the steady state in similar size 
microchannels is around 30 minutes [18]. Other groups work with high complex and expensive set-ups to reach similar flow control results.

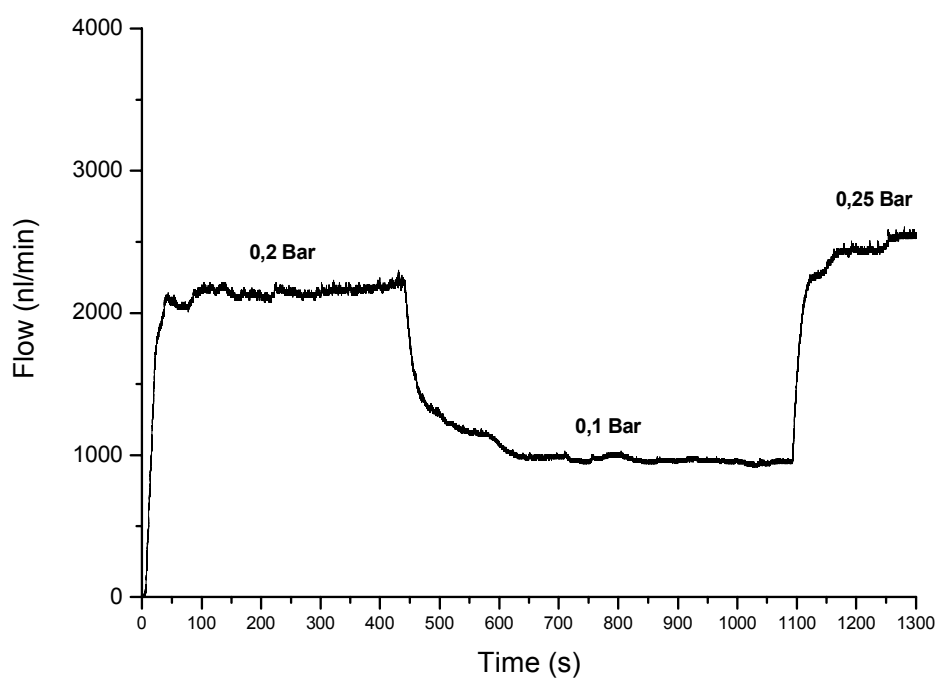

Figure 12. Flow control using switching pressures in a $150 \mu \mathrm{m}$ width and $18 \mu \mathrm{m}$ height microchannel

Further work will be performed in our labs to control flows at different working pressures and channel sizes.

\section{CONCLUSIONS}

A novel polymeric CMOS compatible microfludic technology for BioMOEMS applications has been studied. Two types of packaging technologies have been presented in order to connect our microfluidic devices to the outside. A novel integrated macro-microfluidic approach was developed. This integrated approach can withstand pressure drops up to 2 $\mathrm{MPa}$ without any liquid leakage. Also, a modular approach was developed which can withstand pressure drops up to 500 $\mathrm{MPa}$. The modular approach allows an easy replacement of the external connections. And could allow plug and play macro-microfluidic connections for different applications.

Flow experiments were conducted in the laminar flow regime in order to study the flow behavior through these novel microchannel networks. While the results conform to macro-scale laws, it is still an important result. It provides a basis from which future work can be undertaken in order to precisely predict where the result moves away from that which can be accurately measured using conventional macro scale laws. In addition, accurate flow control at low working pressures was demostrated. Fast flow response to switching pressures has been obtained using a pressure-driven system.

These microfluidic and packaging technologies has a wide range of possibilities due to the high quality of the microfluidic networks and the robustness of the final packaged Microsystems. These technologies will allow the integration and packaging of complex microfluidic systems with different BioMOEMS and CMOS devices.

Further investigation is been performed in order to integrate this microfluidic technology with different biosensor micro and nanodevices.

\section{ACKNOWLEDGEMENTS}

This research is sponsored by the Basque and Spanish Governments, under the Torres Quevedo Spanish Fellowship for industrial research and the strategic research program on micro and nanotechnologies (MICROGUNE). 


\section{REFERENCES}

[1] Manz, A.; Graber, N.; Widmer, H. M. Sens. Actuators 1990, B1,244-248.

[2] Darwin R. Reyes, Dimitri Iossifidis, Pierre-Alain Auroux and Andreas Manz.Micro Total Analysis Systems. 1. Introduction, Theory, and Technology. Anal. Chem. 2002, 74, 2623-2636

[3] Pierre-Alain Auroux, Dimitri Iossifidis, Darwin R. Reyes and Andreas Manz. Micro Total Analysis Systems.2. Analitical standard operations and applications. Anal. Chem. 2002, 74, 2637-2652

[4] Peter Gravensen, Jens Branebjerg and Ole Sondergard Jensen. Microfluidics-a review. J. Micromech. Miocroeng. 3 (1993) 168-182.

[5] Brody, J. P.; Yager, P.; Goldstein, R. E.; Austin . H. Biotechnology at Low Reynolds numbers. Biophys. J. 1996, 71, 3430-3441.

[6] Peter Wilding, Joseph Pfahler, Haim H. Bau, Jay N. Zemel and Larry J. Kricka. Manipulation and Flow of Biological Fluids in Straight channels micromachined in Silicon. Clin. Chem. 40/1, 43-47 (1994) Automation and Analytical Tecniques.

[7] X.F. Peng, G. P. Peterson, B. X. Wang. Frictional flow characteristics of water flowing through rectangular microchannels. Experimental Heat Transfer 7 (1994) 249-264

[8] Peng XF and Peterson GP 1996 Convective heat transfer and flow friction for water flow in microchannel structure Int. J. Heat Mass Transfer 39 2599-2608

[9] Mala et al. Flow characteristics of water through a microchannel between two parallel plates with electokinetic effects. Int J. Heat and Fluid Flow 18: 489-496, 1997

[10] S.M. Flockhart, R.S. Dhariwal. Experimental and numerical investigation into the flow characteristics of channels etched in <100> silicon. Transactions of the ASME: J. Fluids Engng 120 (1998) 291-295

[11] G.M. Mala, D. Li. Flow characteristics of water in microtubes. Int. J. Heat and Fluid Flow 20 (1999) $142-148$.

[12] Qu W, Mala G M and Li D. Pressure driven water flows in trapezoidal silicon microchannels Int. J. Heat Mass Transfer 43 353-364, 2000

[13] Dongquin Li. Electro viscous effects on pressure-driven liquid flow in microchannels.Colloids and Surfaces A: Physicochemical and engineering Aspects 195 (2001) 35-37

[14] Hojoon Park, James Jungh Park, Sang Young Son, Geunbae Lim, Inseob Song .Fabrication of a microchannel integrated with inner sensors and analysis of its laminar flow characteristics. Sensors and Actuatros A (2003) 317-329

[15] Junemo Koo and Clememt Kleinstreuer . Liquid flow in microchannels: experimental observations and computional analyses of microfluidic effects. J. Micromech. Microeng. 13 (2003) 568-579 (2003)

[16] Shou-Shing Hsieh, Chih-Yi Lin, Chin-Feng Huang and Huang-Hsiu Tsai. Liquid flow in a microchannel. J. Micromech. Microeng. 14 (2004) 436-445.

[17] B.J. Kim, Y Z Liu and HJ Sung. Micro PIV measurements of two fluid flow with different refractive indices. Meas. Sci. Technol. 15 (2004) 1097-1103.

[18] K. V. Sharp. Experimental investigation of liquid and particle-laden flows in microtubes. PhD. Thesis. University of Illinois at Urbana-Champaign, 2001.

[19] F J Blanco, M Agirregabiria, J Garcia, J Berganzo, M Tijero, M T Arroyo, J M Ruano, I Aramburu and Kepa Mayora. A Novel low temperature CMOS compatible Full wafer bonding process for the fabrication of 3D embedded microchanels using SU-8 Photoresist. Invited paper. Proceedings of SPIE Vol. 5276 (2004) 131-142 2003 Microelectronics and MEMS International Conference. 12-14 December 2003 (Perth,Australia)

[20] F J Blanco, M Agirregabiria, J Garcia, J Berganzo, M Tijero, M T Arroyo, J M Ruano, I Aramburu and Kepa Mayora. Novel three dimensional embedded SU-8 microchannels fabricated using a low temperature full wafer adhesive bonding. Journal of Micromechanics and Microengeeniring 14 (2004) 1047-1056.

[21] M. Agirregabiria, F. J. Blanco, J. Berganzo, M. T. Arroyo, A. Fullaondo, K. Mayora and J. M. Ruano-LÛpez. Fabrication of SU-8 multilayer microstructures based on successive CMOS compatible adhesive bonding and releasing steps. Lab chip (in press)

[22] Carl K. Fredickson and Z. Hugh Fan . Macro-to-micro interfaces for microfluidic devices. Lab chip, 2004, 4, 526533.

[23] C. Gartner, H. Becker, B. Anton, A. O. Oneill and O. Rotting. Proc. SPIE 2003, 4982, 99-104

[24] C. Garner, H. Becker, B. Anton and O. Rotting. Proc. SPIE, 2004. 5345, 159-162

[25] X.B. Chen and J Kai. Modelling of positive-Displacement Fluid Dispensing Processes. IEEE transactions on Electronics Packaging Manufacturing. July 2004. Vol. 27, N3, pp 157-163

[26] V. Nittis, R. Fortt, C.H. Legge and A.J. Mello, Lab Chip, 2001,1, 128-152. 
[27] N. Goedecke, B. McKenna, S. El-Difrawy, L. Carey, P. Matsudaira and D. Ehrlich, Electrophoresis, 2004, 25, 16781686.

[28] H. Chen, D. Acharya, A. Gajraj and J.-C. Melners, Robust interconnects and packaging for microfluidic elastomeric chips Anal Chem., 2003, 75, 5287-5291 